\title{
Metformin protects against insulin resistance induced by high uric acid in cardiomyocytes via AMPK signaling pathways in vitro and in vivo
}

\section{Zhenyu Jiao}

Beijing Chao-Yang Hospital: Beijing Chaoyang Hospital

\section{Yingqun Chen}

Peking University Shenzhen Hospital

\section{Yang Xie}

Shantou University Second Affiliated Hospital Department of Cardiothoracic Surgery

\section{Yanbing Li}

Beijing Chao-Yang Hospital: Beijing Chaoyang Hospital

\section{Zhi Li ( $\nabla$ lizhi519@126.com )}

Shantou University Second Affiliated Hospital Department of Cardiothoracic Surgery

\section{Research}

Keywords: metformin, high uric acid, insulin resistance, AMPK, cardiomyocyte

Posted Date: March 10th, 2021

DOl: https://doi.org/10.21203/rs.3.rs-300030/v1

License: (c) (1) This work is licensed under a Creative Commons Attribution 4.0 International License. Read Full License 


\section{Abstract}

\section{Background}

High uric acid (HUA) is associated with insulin resistance and abnormal glucose metabolism in cardiomyocytes. Metformin is a recognized agonist of AMP-activated protein kinase (AMPK) and an antidiabetic drug widely used for type 2 diabetes. It can play a cardioprotective role in many pathways. We investigated whether metformin protects against HUA-induced insulin resistance and abnormal glucose metabolism in cardiomyocytes.

Methods

We exposed primary cardiomyocytes to HUA, and cellular glucose uptake was quantified by measuring the uptake of 2-NBDG, a fluorescent glucose analog, after insulin excitation.

Results

Treatment with metformin $(10 \mu \mathrm{mol} / \mathrm{L})$ protected against HUA-inhibited glucose uptake induced by insulin in primary cardiomyocytes, as shown by fluorescence microscopy and flow cytometry analysis. HUA directly inhibited the phosphorylation of Akt and the translocation of glucose transporter type 4 (GLUT4) induced by insulin, which was blocked by metformin. Metformin promoted phosphorylation of AMPK, renewed HUA-inhibited glucose uptake induced by insulin and protected against insulin resistance in cardiomyocytes. As a result of these effects, in a mouse model of acute hyperuricemia, metformin improved insulin tolerance and glucose tolerance, accompanied by increased AMPK phosphorylation, Akt phosphorylation and translocation of GLUT4 in myocardial tissues. As expected, AICAR, another AMPK activator, had equivalent effects to metformin, demonstrating the important role of AMPK activation in protecting against insulin resistance induced by HUA in cardiomyocytes. Metformin protects against insulin resistance induced by HUA in cardiomyocytes and improves insulin tolerance and glucose tolerance in an acute hyperuricemic mouse model, along with the activation of AMPK.

Conclusions

Consequently, metformin may be an important potential new treatment strategy for hyperuricemia-related cardiovascular disease.

\section{Introduction}

Insulin resistance usually refers to a clinicopathological condition characterized by an impaired ability of insulin to stimulate glucose uptake and by glucose intolerance [1]. Moreover, insulin resistance is closely related to several cardiovascular diseases, such as myocardial infarction, hypertension and heart failure [2-4]. Clinical follow-up studies have demonstrated that elevated serum uric acid is a strong independent risk factor for insulin resistance [5], and ours and others' previous studies have revealed that high uric acid (HUA) evokes insulin resistance in cardiomyocytes, adipocytes, muscle and liver [6-9]. 
Adenosine $5^{\prime}$-monophosphate-activated protein kinase (AMPK) is an essential metabolic regulator for energy sensing and has been well documented [10]; AMPK is expressed specifically in various tissues and cells, including cardiomyocytes [11], and plays a crucial role in regulating energy metabolism and energy homeostasis under stress conditions. AMPK can be activated by various conditions. Activation of AMPK phosphorylation regulates a series of metabolic steps by inducing the ATP generation system (such as glycolysis and fatty acid oxidation) and inhibiting the ATP consumption system (such as the synthesis of fatty acids). In addition, AMPK is very beneficial for improving insulin sensitivity in many cells and tissues. Our previous data demonstrated that AMPK is activated by HUA via oxidative stress in $\beta$ cells [12]. In our previous study, HUA induced oxidative stress and played a critical role in the development of insulin resistance and its potential role in the pathogenesis of metabolic stress in cardiomyocytes [6]. Moreover, phosphorylated Akt-mediated insulin signal transduction and translocation of glucose transporter type 4 (GLUT4) were suppressed by HUA and were related to insulin resistance and impaired glucose uptake in cardiomyocytes [6].

Metformin, well known as an AMPK activator and as an antidiabetic medicine, is widely used and has various pharmacologic effects, including promoting glucose uptake and utilization, enhancing insulin sensitivity and improving hyperlipidemia [13]. Major advances in the scientific understanding of metformin action have focused on the discovery that metformin leads to the activation of phosphoAMPK. This activation of phospho-AMPK appears to be more associated with changes in phosphocreatine levels and the AMP/ATP ratio. The AMPK system acts as an energy-sparing sensor, and therefore AMPK is a good mediator of metformin activity on heightened glucose uptake and the improved cellular status of energy that follows glucose uptake.

In our previous study, we found that HUA induced insulin resistance and suppressed glucose uptake in cardiomyocytes in vitro and in vivo [6]. In the present study, we assessed whether there are positive effects of metformin's performance on glucose uptake inhibited by HUA in cardiomyocytes and whether treatment with metformin can either circumvent or reverse insulin resistance induced by HUA. At the cellular level, insulin resistance can be induced by prolonged incubation with HUA in cardiomyocytes. The impacts of HUA may be related to reductions in insulin signaling events at the level of phosphatidylinositol 3-kinase activation of Akt.

Previous studies have demonstrated that metformin can protect the heart by inhibiting apoptosis, autophagy, inflammation and other pathways via AMPK $[9,11,14]$. However, whether metformin protects against insulin resistance induced by HUA in cardiomyocytes remains unknown. Therefore, we determined whether metformin activation of the AMPK-dependent pathway can protect against insulin resistance induced by HUA in cardiomyocytes.

\section{Methods And Materials}

Reagents 
Uric acid ( $15 \mathrm{mg} / \mathrm{dl}$, final concentration), metformin, insulin (100 nM, final concentration) and compound $\mathrm{C}(20 \mu \mathrm{M}$, final concentration) were purchased from Sigma (St. Louis, MO, USA). N-(7-Nitrobenz-2-oxa-1,3diazol-4-yl)amino]-2-deoxy-d-glucose (2-NBDG, $100 \mu \mathrm{M}$, final concentration) and anti-IRS1 (Ser307) and anti-phospho-IRS1 (Ser307) antibodies were purchased from Invitrogen (Carlsbad, CA, USA). 5-Amino-4imidazole-1- $\beta$-D-carboxamide ribofuranoside (AICAR, $500 \mu \mathrm{M}$, final concentration) was purchased from MedChemExpress (MCE LLC, USA). A rabbit anti-GAPDH antibody was purchased from Abcam (Abcam, USA). Anti-Akt (Ser473) and anti-phospho-Akt (Ser473) antibodies were purchased from Bioworld (St. Louis Park, USA). Anti-AMPK (Thr172) and anti-phospho-AMPK (Thr172) antibodies were purchased from Cell Signaling Technology (CST, Beverly, MA, USA). Cardiomyocytes were cultured in serum-free media for 24 hours and then incubated in the presence of $15 \mathrm{mg} / \mathrm{dl} \mathrm{HUA}$ for 24 hours. Cardiomyocytes were pretreated with either metformin ( 0 to $20 \mu \mathrm{mol} / \mathrm{L}$ ) or AICAR, an AMPK activator $(500 \mu \mathrm{mol} / \mathrm{L})$, for 60 minutes before the addition of HUA. Other cells were preincubated with compound $C$ for 6 hours before the addition of either metformin or AICAR. Then, 2-NBDG uptake was analyzed in cardiomyocytes.

were from Millipore (Billerica, MA).

\section{Cell culture}

Cellular studies were carried out on primary cardiomyocytes isolated from C57BL/6 neonatal mice according to a previous protocol [15]. Cardiomyocytes were incubated with Dulbecco's modified Eagle medium (DMEM) or low-glucose minimum essential medium eagle (MEM) containing L-glutamine (2 $\mathrm{mM})$, streptomycin $(100 \mathrm{mg} / \mathrm{ml})$, penicillin $\mathrm{G}(100 \mathrm{U} / \mathrm{ml})$ and $10 \%$ fetal bovine serum. For all experiments, the cardiomyocytes were seeded into 6 -well plates at a density of $2.0 \times 10^{5}$ cells $/ \mathrm{ml}$. Primary cardiomyocytes were incubated in a $37^{\circ} \mathrm{C}$ incubator with $95 \%$ air and $5 \% \mathrm{CO}_{2}$.

\section{Uptake of 2-NBDG glucose was measured by fluorescence microscopy and flow cytometry}

A fluorescent glucose analog, 2-NBDG, glucose uptake, was measured by fluorescence microscopy and flow cytometry in cardiomyocytes to assess glucose uptake.

Briefly, for fluorescence microscopy, the cells were incubated in fetal bovine serum-free medium for 24 hours. The culture medium was replaced by Krebs-Ringer-Bicarbonate buffer containing insulin (100 nM) and 2-NBDG $(100 \mu \mathrm{M})$ at $37^{\circ} \mathrm{C}$ for 30 minutes and analyzed at excitation and emission wavelengths of 488 and $525 \mathrm{~nm}$, respectively.

For flow cytometry, the cells were fed low-glucose DMEM without fetal bovine serum for 24 hours. The culture medium was then replaced with Krebs-Ringer-Bicarbonate (KRB) buffer containing 2-NBDG (100 $\mu \mathrm{M})$ and insulin $(100 \mathrm{nM})$ for 30 minutes at $37^{\circ} \mathrm{C}$ in a $\mathrm{CO}_{2}$ incubator. For flow cytometry-based 2-NBDG glucose uptake assays, after treatment, 2-NBDG was washed out of the culture medium, and uptake of 2NBDG was assessed by flow cytometry with a fluorometer at an excitation wavelength of $488 \mathrm{~nm}$ and an 
emission wavelength of $525 \mathrm{~nm}$. For each sample, 20,000 cells in each well were obtained in the FSC $x$ SSC plots.

\section{Animal experiments}

The protocol was unanimously affirmed by the Shantou University Animal Experiments Ethics Committee. All methods were performed in accordance with the relevant guidelines.

C57BL/6J male mice (8 weeks old) were obtained from Vital River Laboratories Animal, Ltd. (Beijing, China) in this study. All animals were housed in the Laboratory Animal Center of Shantou University Medical College. The animals were housed in cages under a 12-hour light-dark cycle and fed water and a normal food diet. Cardiac myocytes and ventricle cardiac tissue were obtained as previously reported [16].

\section{Experimental acute hyperuricemia mouse model establishment and metformin treatment}

Ten-week-old male mice were randomly divided into 3 groups ( $n=6$ each) for treatment: the control, $\mathrm{HU}$ (hyperuricemia) and HU-Met (metformin) groups. For the HU group, following an 18-hour overnight fast, the mice were injected intraperitoneally (i.p.) with $300 \mathrm{mg} / \mathrm{kg}$ potassium oxonate and intragastrically with $500 \mathrm{mg} / \mathrm{kg}$ hypoxanthine to create the model of acute hyperuricemia for 1-2 hours. The mice in the HUMet group were administered $250 \mathrm{mg} / \mathrm{kg} /$ day metformin in drinking water for 14 consecutive days [17]. The quantity of medicine was based on body weight levels before each dose. The level of serum uric acid was determined by the phosphotungstic acid method at different times [18]. Insulin tolerance tests (ITTs) and glucose tolerance tests (GTTs) were performed as described previously [8]. For the control group and $\mathrm{HU}$ group, the mice were sacrificed by $\mathrm{CO}_{2}$ inhalation 10 minutes after injection with $2 \mathrm{U} / \mathrm{kg}$ insulin.

Cardiac muscle tissue was excised and stored in liquid nitrogen immediately.

\section{Western blot}

Cardiomyocytes were washed twice with cold PBS, harvested, lysed in ice-cold lysis buffer $(\mathrm{pH}=7.4,100$ $\mathrm{mM} \mathrm{KCl}, 10 \mathrm{mM}$ HEPES, $1.5 \mathrm{mM} \mathrm{MgCl}$ ), sonicated and homogenized in RIRP buffer. After centrifugation, a Pierce BCA protein assay kit was used to determine the protein concentration. Equal concentrations of soluble lysate protein $(50 \mathrm{mg}$ ) were added to a $12 \%$ SDS-PAGE Bis-Tris gel, and the protein was transferred to immunoblot polyvinylidene fluoride (PVDF) membranes (Bio-Rad, USA). Membranes were incubated and blocked overnight at $4^{\circ} \mathrm{C}$ with antibodies against p-AMPK/AMPK, p-Akt/Akt and GAPDH. Then, the membranes were incubated with anti-rabbit horseradish peroxidase-conjugated secondary antibodies (1:10,000 dilution). The immunoreactive bands were analyzed with an enhanced chemiluminescence substrate kit (Biological Industries, BI, Israel).

\section{Statistical analysis}

Data are presented as the means \pm SD. Significant differences between the means were analyzed by a Student's t-test. Significant differences were evaluated using one-way analyses of variance (ANOVA), and $P$ values less than 0.05 were considered to be statistically significant. 


\section{Results}

\section{Metformin stimulated the phosphorylation of AMPK and attenuated insulin resistance induced by HUA in cardiomyocytes}

We first wanted to determine the most suitable exposure period and concentration of metformin for the activation of phospho-AMPK in cardiomyocytes. Treatment with metformin (using the most suitable concentration and exposure period of $10 \mu \mathrm{M}$ for 60 minutes) stimulated AMPK phosphorylation in cardiomyocytes in a dose- and time-dependent manner (Fig. 1A and 1B). Moreover, 2-NBDG glucose uptake was decreased significantly in the presence of HUA $(15 \mathrm{mg} / \mathrm{dl})$ for 24 hours in cardiomyocytes, as shown by the flow cytometry assay. These results demonstrated that HUA inhibited 2-NBDG glucose uptake induced by insulin and caused insulin resistance in primary cardiomyocytes, but this change was attenuated in a dose- and time-dependent manner by treatment with metformin (Fig. 1C).

\section{Metformin attenuated insulin resistance induced by HUA via AMPK signaling pathways in cardiomyocytes}

To investigate whether metformin attenuates HUA-induced insulin resistance via AMPK signaling pathways in cardiomyocytes, cardiomyocytes were pretreated with metformin $(10 \mu \mathrm{M})$ for 60 minutes and/or compound-C $(20 \mu \mathrm{M})$, a specific AMPK inhibitor, for 6 hours before exposure to HUA. Under these conditions, the effect of metformin on 2-NBDG glucose uptake was blunted by cotreatment with compound C (Fig. 2A-2C).

To confirm the role of AMPK signaling pathways in metformin attenuation of HUA-induced insulin resistance in cardiomyocytes, cells were pretreated with another AMPK activator, AICAR $(500 \mu \mathrm{M})$, for 60 minutes before exposure to HUA. We found that AICAR had an effect similar to metformin on glucose (2NBDG) uptake after exposure to HUA (Fig. 3A-3C). These findings demonstrated that activation of AMPK signaling pathways protected against insulin resistance induced by HUA in cardiomyocytes.

Metformin increased phospho-AMPK, phospho-Akt and translocation of GLUT4 but had no effect on phospho-IRS1 expression in cardiomyocytes

AMPK is a well-known master energy sensing regulator. Furthermore, the Akt family of Thr/Ser protein kinases is of critical importance with regard to cardiac metabolism and growth in insulin signal transduction. Consequently, to further inspect the molecular mechanism of the AMPK and Akt signaling pathways, we first examined whether metformin activated AMPK in cardiomyocytes. In cardiomyocytes, metformin enhanced the phosphorylation of AMPK, and this phosphorylation was blocked by compound C, a specific AMPK inhibitor (Fig. 4A). 
To test whether metformin also activated the expression of phospho-Akt and translocation of GLUT4 in cardiomyocytes, we determined phospho-Akt expression and translocation of GLUT4 by performing western blot analyses in cardiomyocytes. We demonstrated that the insulin-induced phosphorylation level of Akt and translocation of GLUT4 were significantly inhibited by HUA (Fig. 4B-4C) but had no effect on phospho-IRS1 (Fig. 4D) expression in cardiomyocytes. Furthermore, we investigated the role of metformin in HUA-mediated inhibition of Akt activation and GLUT4 translocation. Metformin did not influence the insulin-induced activation of Akt kinase phosphorylation or translocation of GLUT4 (Fig. 4B-4C). However, insulin-induced activation of Akt kinase phosphorylation and translocation of GLUT4 were depressed by HUA, which was attenuated significantly by metformin (Fig. 4B-4C). Nevertheless, the effect of metformin on Akt phosphorylation and translocation of GLUT4 were depressed by HUA, which was significantly offset by compound C, an AMPK inhibitor. As expected, we found that AICAR had an effect similar to metformin on phospho-AMPK, phospho-Akt and translocation of GLUT4 after exposure to HUA (Fig. 4A4C). These results demonstrated a profound effect of metformin on downstream targets of the insulin signaling pathway via AMPK.

\section{Effect of metformin in a hyperuricemia mice model Effect of metformin on insulin resistance induced by hyperuricemia in a mouse model}

As expected, the levels of serum uric acid in the acute hyperuricemia mouse model were significantly higher than those before hyperuricemia induction (115.69 \pm 18.32 vs $39.71 \pm 4.43 \mathrm{mg} / \mathrm{l})$, which was consistent with primary hyperuricemia patients [19]. Our acute hyperuricemia mouse model also indicated an impaired glucose tolerance test (Fig. 5A) and insulin tolerance test (Fig. 5B) at 15 and 30 minutes after insulin or glucose injection with insulin resistance. Treatment with metformin significantly decreased the level of serum glucose in the glucose tolerance test and insulin tolerance test compared with the hyperuricemia mouse model (Fig. 5A and 5B). Thus, these findings demonstrate that metformin protects against hyperuricemia-induced insulin resistance in a mouse model.

Effect of metformin on AMPK, IRS1 phosphorylation, Akt phosphorylation and translocation of GLUT4 in cardiac tissues from the acute hyperuricemic mouse model

To examine the molecular mechanisms by which metformin protects against hyperuricemia-induced insulin resistance in cardiomyocytes in an acute hyperuricemic mouse model, we investigated the effect of metformin on AMPK, IRS1 phosphorylation, Akt phosphorylation and GLUT4 translocation in cardiac tissues from the acute hyperuricemic mouse model. The hyperuricemic mice were injected with $2 \mathrm{U} / \mathrm{kg}$ insulin, and after 10 minutes, the cardiac tissues were isolated and obtained. Metformin increased the phosphorylation level of AMPK in mouse cardiac tissues (Fig. $5 \mathrm{C}$ ). The western blot analysis results demonstrated a significant reduction in the level of phospho-AKT and translocation of GLUT4 in the hyperuricemic mouse model, while metformin reversed these changes (Fig. 5E-5F) but had no effect on 
phospho-IRS1 (Fig. 5D). These results demonstrate that metformin protects against hyperuricemiainduced insulin resistance in cardiomyocytes via AMPK signaling pathways in vivo.

\section{Discussion}

To the best of our knowledge, this is the first study to clearly illustrate that metformin, which is used worldwide as an antidiabetic medicine, protects against insulin resistance in cardiomyocytes induced by HUA and increases AMPK activation. Of course, we and others have previously shown that HUA induces insulin resistance in various cells, including cardiomyocytes, skeletal muscle cells and HepG2 cells [6-9]. Nevertheless, it has been unclear whether AMPK can regulate insulin resistance induced by HUA in cardiomyocytes in vitro and in vivo. Therefore, we used neonatal mouse primary cardiomyocytes and an acute hyperuricemia mouse model, which is regarded as similar to humans with primary hyperuricemia and can be utilized in translational research for human hyperuricemia.

Hyperuricemia is strongly associated with cardiovascular risk and a poor outcome in a variety of cardiovascular disease states, such as coronary artery disease, hypertension and heart failure [20-24]. However, the underlying mechanisms to explain this association have been nonexistent. In our previous study [6], we explored the impact of HUA on glucose uptake and insulin resistance in primary cardiomyocytes and found that HUA induces oxidative stress, which plays a crucial role in the progression of insulin resistance in cardiomyocytes. Moreover, recent studies have confirmed insulin resistance as a powerful independent predictor of mortality and morbidity in patients with heart failure [25-26]. These studies demonstrated that therapeutically targeting impaired insulin sensitivity may potentially be favorable for patients with chronic heart failure. To determine whether metformin protects against insulin resistance induced by HUA in cardiomyocytes, we exposed primary cardiomyocytes to HUA, pretreated them with metformin and then quantified the uptake of glucose with 2-NBDG, a fluorescent glucose analog, after insulin stimulation. We found that treatment with metformin may protect cardiomyocytes from HUA and inhibit insulin-induced glucose uptake in cardiomyocytes. These results suggested that HUA resulted in insulin resistance in cardiomyocytes, but this change was weakened by pretreatment with metformin.

Metformin is well known to activate AMPK, which is expressed in a variety of tissues and cells, including cardiomyocytes, and plays a pivotal role in the regulation of cellular energy metabolism under stress conditions [14]. Previous studies have shown that metformin reduces long-term and high insulin-induced insulin resistance in cardiomyocytes [27], and AMPK has been shown to protect against insulin resistance in skeletal muscle cells through restoration of GLUT4 translocation [28]. Consistent with the findings of these previous studies, we found that metformin could improve HUA-induced insulin resistance in primary cardiomyocytes. As expected, this change was blocked by compound C, an AMPK inhibitor, indicating that AMPK activation was responsible for the suppression of insulin resistance in cardiomyocytes. In addition, using an acute hyperuricemic mouse model, our study indicated that metformin improved the progression of insulin resistance induced by HUA, as shown by the glucose tolerance test (Fig. 5A) and insulin tolerance test (Fig. 5B). 
Interestingly, another activator of AMPK, AICAR, had almost the same effects as metformin, suggesting that activation of AMPK phosphorylation promoted the observed protective effect of insulin resistance in cardiomyocytes. In fact, AICAR has been demonstrated to protect against myocardial ischemiareperfusion injury after myocardial infarction in animals and humans [29-30]. However, what procedures following AMPK pathway activation are involved in cardioprotection?

The first possibility is the improvement of the insulin-activated Akt pathway, which is inhibited by HUA. A previous study demonstrated that phosphorylation of Akt regulates cell survival, growth and metabolism. Additionally, cardiomyocyte metabolism and growth are coordinated by the integration of intracellular and extracellular signals [31]. Furthermore, activation of Akt phosphorylation, a key protein kinase of insulin-induced glucose uptake, modulates glucose uptake stimulated by insulin in cardiomyocytes [6]. In the present study, we found that HUA could strongly inhibit Akt phosphorylation, translocation of GLUT4 and 2-NBDG glucose uptake induced by insulin in primary cardiomyocytes. Our findings suggest that activators of AMPK, such as metformin and AICAR, could prevent HUA-inhibited Akt phosphorylation, GLUT4 translocation and HUA-inhibited 2-NBDG glucose uptake in cardiomyocytes. Moreover, an acute hyperuricemia mouse model demonstrated inhibited Akt phosphorylation with insulin resistance and glucose intolerance.

Another possibility is the metabolic effects of AMPK phosphorylation activation. Metformin has been demonstrated to activate the phosphorylation of AMPK in cardiomyocytes and mouse cardiac tissues. In fact, a recent study suggested that short-term treatment with metformin protects against myocardial ischemia-reperfusion injury via the AMPK signaling pathway after myocardial infarction in mice [11]. Therefore, AMPK has been considered to have various cardioprotective effects in animals and humans. Improvement in AMPK production by metformin may have mitigated the progression of insulin resistance induced by HUA. Both AICAR and metformin are reported to enhance glucose uptake in skeletal muscle cells and heart muscle cells $[9,31]$. Consistent with these reports, in the HUA plus insulin group, we found that pretreatment with metformin and AICAR nearly reverted glucose uptake to the level of the control group in cardiomyocytes. Therefore, the possibility exists that the AMPK-induced uptake of glucose triggers improvement in insulin resistance, which is induced by HUA, followed by the restitution of the metabolic switch.

The decreased Akt phosphorylation and translocation of GLUT4 in the HUA group were reversed in both the HUA plus AICAR group or the HUA plus metformin group, demonstrating that the activation of AMPK protects the insulin signaling pathway of Akt against inhibition by HUA in both the HUA plus AICAR group or the HUA plus metformin group.

\section{Conclusions}

Our findings illustrated that metformin protects against the progression of insulin resistance induced by HUA in cardiomyocytes and activates phospho-AMPK. These findings show an interaction between activation of AMPK induced by metformin and insulin resistance induced by HUA that could be important 
to our understanding of the potentiating effects of metformin on patients with insulin resistance and hyperuricemia in clinical trials. Therefore, metformin may provide a new treatment strategy for hyperuricemia-related cardiovascular disease.

\section{Abbreviations}

HUA: high uric acid; AMPK: AMP-activated protein kinase; GLUT4: glucose transporter type 4; 2-NBDG: N(7-Nitrobenz-2-oxa-1,3-diazol-4-yl)amino]-2-deoxy-d-glucose; AICAR: 5-Amino-4-imidazole-1- $\beta$-Dcarboxamide ribofuranoside; IRS1: insulin receptor substrate 1; DMEM: Dulbecco's modified Eagle medium; MEM: minimum essential medium eagle; KRB: Krebs-Ringer-Bicarbonate; ITTs: Insulin tolerance tests;GTTs: glucose tolerance tests; ANOVA: one-way analyses of variance.

\section{Declarations}

\section{Ethics approval and consent to participate}

The protocol was unanimously affirmed by the Shantou University Animal Experiments Ethics Committee (Approval number: SUMC2017-049). All methods were performed in accordance with the relevant guidelines.

\section{Consent for publication}

Not applicable.

\section{Availability of data and materials}

All data generated or analyzed during this study are included in this published article.

\section{Competing interests}

The authors declare no conflict of interest. All authors read and approved the final manuscript.

\section{Funding}

This work was supported by grants from the Guangdong Basic and Applied Basic Research Foundation of China (2018A030307056), the Guangdong Medical Science and Technology Research Fund Project of China (A2020159) and the Shantou Science and Technology Plan Project Foundation of China ([2018]155).

\section{Author Contributions}

Conceived and designed the experiments: Yanbing Li \& Zhi Li. Performed the experiments: Zhenyu Jiao \& Yingqun Chen. Analyzed the data: Yang Xie. Contributed reagents/materials/analysis tools: Yang Xie. Wrote the paper: Zhenyu Jiao \& Zhi Li. All authors reviewed the manuscript. 


\section{Acknowledgements}

We would like to thank central laboratory of Second Affiliated Hospital of Shantou University Medical College.

\section{References}

1 Tan Y, Ichikawa T, Li J, Si Q, Yang H, Chen X, Goldblatt CS, Meyer CJ, Li X, Cai L, Cui T. Diabetic downregulation of Nrf2 activity via ERK contributes to oxidative stress-induced insulin resistance in cardiac cells in vitro and in vivo. Diabetes. 2011 Feb;60(2):625-33. doi: 10.2337/db10-1164. PMID: 21270272 ; PMCID: PMC3028364.

2 Kanellakis S, Mavrogianni C, Karatzi K, Lindstrom J, Cardon G, lotova V, Wikström K, Shadid S, Moreno LA, Tsochev K, Bíró É, Dimova R, Antal E, Liatis S, Makrilakis K, Manios Y. Development and Validation of Two Self-Reported Tools for Insulin Resistance and Hypertension Risk Assessment in A European Cohort: The Feel4Diabetes-Study. Nutrients. 2020 Mar 30;12(4):960. doi: 10.3390/nu12040960. PMID: 32235566; PMCID: PMC7230581.

3 Wiebe N, Stenvinkel P, Tonelli M. Associations of Chronic Inflammation, Insulin Resistance, and Severe Obesity With Mortality, Myocardial Infarction, Cancer, and Chronic Pulmonary Disease. JAMA Netw Open. 2019;2(8):e1910456. doi:10.1001/jamanetworkopen.2019.10456

4 Uddin GM, Zhang L, Shah S, Fukushima A, Wagg CS, Gopal K, Al Batran R, Pherwani S, Ho KL, Boisvenue J, Karwi QG, Altamimi T, Wishart DS, Dyck JRB, Ussher JR, Oudit GY, Lopaschuk GD. Impaired branched chain amino acid oxidation contributes to cardiac insulin resistance in heart failure. Cardiovasc Diabetol. 2019 Jul 5;18(1):86. doi: 10.1186/s12933-019-0892-3. PMID: 31277657; PMCID: PMC6610921.

5 Han T, Lan L, Qu R, Xu Q, Jiang R, Na L, Sun C. Temporal Relationship Between Hyperuricemia and Insulin Resistance and Its Impact on Future Risk of Hypertension. Hypertension. 2017 Oct;70(4):703-711. doi: 10.1161/HYPERTENSIONAHA.117.09508. Epub 2017 Aug 14. PMID: 28808071.

6 Zhi L, Yuzhang Z, Tianliang H, Hisatome I, Yamamoto T, Jidong C. High Uric Acid Induces Insulin Resistance in Cardiomyocytes In Vitro and In Vivo. PLoS One. 2016 Feb 2;11(2):e0147737. doi: 10.1371/journal.pone.0147737. PMID: 26836389; PMCID: PMC4737875.

7 Su M, Sun L, Li W, Liu H, Liu Y, Wei Y, Yuan Y, Zheng L, Yin S, Dai C, Zhao C, Pan Z, Li Y. Metformin alleviates hyperuricaemia-induced serum FFA elevation and insulin resistance by inhibiting adipocyte hypertrophy and reversing suppressed white adipose tissue beiging. Clin Sci (Lond). 2020 Jun 26;134(12):1537-1553. doi: 10.1042/CS20200580. PMID: 32556103.

8 Zhu Y, Hu Y, Huang T, Zhang Y, Li Z, Luo C, Luo Y, Yuan H, Hisatome I, Yamamoto T, Cheng J. High uric acid directly inhibits insulin signalling and induces insulin resistance. Biochem Biophys Res Commun. 2014 May 16;447(4):707-14. doi: 10.1016/j.bbrc.2014.04.080. Epub 2014 Apr 21. PMID: 24769205. 
9 Yuan H, Hu Y, Zhu Y, Zhang Y, Luo C, Li Z, Wen T, Zhuang W, Zou J, Hong L, Zhang X, Hisatome I, Yamamoto T, Cheng J. Metformin ameliorates high uric acid-induced insulin resistance in skeletal muscle cells. Mol Cell Endocrinol. 2017 Mar 5;443:138-145. doi: 10.1016/j.mce.2016.12.025. Epub 2016 Dec 30. PMID: 28042024.

10 Toyama EQ, Herzig S, Courchet J, Lewis TL Jr, Losón OC, Hellberg K, Young NP, Chen H, Polleux F, Chan DC, Shaw RJ. Metabolism. AMP-activated protein kinase mediates mitochondrial fission in response to energy stress. Science. 2016 Jan 15;351(6270):275-281. doi: 10.1126/science.aab4138. PMID: $26816379 ;$ PMCID: PMC4852862.

11 Hua J, Liu Z, Liu Z, An D, Lai W, Zhan Q, Zeng Q, Ren H, Xu D. Metformin Increases Cardiac Rupture After Myocardial Infarction via the AMPK-MTOR/PGC-1a Signaling Pathway in Rats with Acute Myocardial Infarction. Med Sci Monit. 2018 Oct 2;24:6989-7000. doi: 10.12659/MSM.910930. PMID: 30275441; PMCID: PMC6180847.

12 Zhang Y, Yamamoto T, Hisatome I, Li Y, Cheng W, Sun N, Cai B, Huang T, Zhu Y, Li Z, Jing X, Zhou R, Cheng J. Uric acid induces oxidative stress and growth inhibition by activating adenosine monophosphate-activated protein kinase and extracellular signal-regulated kinase signal pathways in pancreatic $\beta$ cells. Mol Cell Endocrinol. 2013 Aug 15;375(1-2):89-96. doi: 10.1016/j.mce.2013.04.027. Epub 2013 May 23. PMID: 23707617.

13 Forslund K, Hildebrand F, Nielsen T, Falony G, Le Chatelier E, Sunagawa S, Prifti E, Vieira-Silva S, Gudmundsdottir V, Pedersen HK, Arumugam M, Kristiansen K, Voigt AY, Vestergaard H, Hercog R, Costea PI, Kultima JR, Li J, Jørgensen T, Levenez F, Dore J; MetaHIT consortium, Nielsen HB, Brunak S, Raes J, Hansen T, Wang J, Ehrlich SD, Bork P, Pedersen O. Corrigendum: Disentangling type 2 diabetes and metformin treatment signatures in the human gut microbiota. Nature. 2017 May 3;545(7652):116. doi: 10.1038/nature22318. Erratum for: Nature. 2015 Dec 10;528(7581):262-266. PMID: 28470190.

14 Sasaki H, Asanuma H, Fujita M, Takahama H, Wakeno M, Ito S, Ogai A, Asakura M, Kim J, Minamino T, Takashima S, Sanada S, Sugimachi M, Komamura K, Mochizuki N, Kitakaze M. Metformin prevents progression of heart failure in dogs: role of AMP-activated protein kinase. Circulation. 2009 May 19;119(19):2568-77. doi: 10.1161/CIRCULATIONAHA.108.798561. Epub 2009 May 4. PMID: 19414638.

15 Yue R, Hu H, Yiu KH, Luo T, Zhou Z, Xu L, Zhang S, Li K, Yu Z. Lycopene protects against hypoxia/reoxygenation-induced apoptosis by preventing mitochondrial dysfunction in primary neonatal mouse cardiomyocytes. PLoS One. 2012;7(11):e50778. doi: 10.1371/journal.pone.0050778. Epub 2012 Nov 30. PMID: 23226382; PMCID: PMC3511264.

16 Wang C, Liu N, Luan R, Li Y, Wang D, Zou W, Xing Y, Tao L, Cao F, Wang H. Apelin protects sarcoplasmic reticulum function and cardiac performance in ischaemia-reperfusion by attenuating oxidation of sarcoplasmic reticulum Ca2+-ATPase and ryanodine receptor. Cardiovasc Res. 2013 Oct 1;100(1):114-24. doi: 10.1093/cvr/cvt160. Epub 2013 Jun 13. PMID: 23771946. 
17 Han X, Tao Y, Deng Y, Yu J, Sun Y, Jiang G. Metformin accelerates wound healing in type 2 diabetic db/db mice. Mol Med Rep. 2017 Dec;16(6):8691-8698. doi: 10.3892/mmr.2017.7707. Epub 2017 Oct 4. PMID: 28990070; PMCID: PMC5779947.

18 Li JM, Zhang X, Wang X, Xie YC, Kong LD. Protective effects of cortex fraxini coumarines against oxonate-induced hyperuricemia and renal dysfunction in mice. Eur J Pharmacol. 2011 Sep;666(1-3):196204. doi: 10.1016/j.ejphar.2011.05.021. Epub 2011 May 23. PMID: 21620826.

19 Nakagawa T, Hu H, Zharikov S, Tuttle KR, Short RA, Glushakova O, Ouyang X, Feig DI, Block ER, Herrera-Acosta J, Patel JM, Johnson RJ. A causal role for uric acid in fructose-induced metabolic syndrome. Am J Physiol Renal Physiol. 2006 Mar;290(3):F625-31. doi: 10.1152/ajprenal.00140.2005. Epub 2005 Oct 18. PMID: 16234313.

20 Tai S, Li X, Zhu Z, Tang L, Yang H, Fu L, Hu X, Fang Z, Zhou S. Hyperuricemia is a Risk Factor for OneYear Overall Survival in Elderly Female Patients with Acute Coronary Syndrome. Cardiovasc Ther. 2020 Feb 22;2020:2615147. doi: 10.1155/2020/2615147. PMID: 32161625; PMCID: PMC7057023.

21 Huang WS, Lin CL, Tsai CH, Chang KH. Association of gout with CAD and effect of antigout therapy on CVD risk among gout patients. J Investig Med. 2020 Jun;68(5):972-979. doi: 10.1136/jim-2019-001140. Epub 2020 Feb 24. PMID: 32098832; PMCID: PMC7306869.

22 Krishnan E, Baker JF, Furst DE, Schumacher HR. Gout and the risk of acute myocardial infarction. Arthritis Rheum. 2006 Aug;54(8):2688-96. doi: 10.1002/art.22014. PMID: 16871533.

23 Anker SD, Doehner W, Rauchhaus M, Sharma R, Francis D, Knosalla C, Davos CH, Cicoira M, Shamim W, Kemp M, Segal R, Osterziel KJ, Leyva F, Hetzer R, Ponikowski P, Coats AJ. Uric acid and survival in chronic heart failure: validation and application in metabolic, functional, and hemodynamic staging. Circulation. 2003 Apr 22;107(15):1991-7. doi: 10.1161/01.CIR.0000065637.10517.A0. Epub 2003 Apr 21. PMID: 12707250.

24 Doehner W, Rauchhaus M, Ponikowski P, Godsland IF, von Haehling S, Okonko DO, Leyva F, Proudler AJ, Coats AJ, Anker SD. Impaired insulin sensitivity as an independent risk factor for mortality in patients with stable chronic heart failure. J Am Coll Cardiol. 2005 Sep 20;46(6):1019-26. doi:

10.1016/j.jacc.2005.02.093. PMID: 16168285.

25 Szabo T, von Haehling S, Habedank D, Rauchhaus M, Lainscak M, Sandek A, Schefold J, Anker SD, Doehner W. Usefulness of minimal modelling to assess impaired insulin sensitivity in patients with chronic heart failure. Int J Cardiol. 2011 Feb 17;147(1):47-51. doi: 10.1016/j.ijcard.2009.07.030. Epub 2009 Sep 5. PMID: 19733925.

26 Yang J, Holman GD. Long-term metformin treatment stimulates cardiomyocyte glucose transport through an AMP-activated protein kinase-dependent reduction in GLUT4 endocytosis. Endocrinology. 2006 Jun;147(6):2728-36. doi: 10.1210/en.2005-1433. Epub 2006 Mar 2. PMID: 16513829. 
27 Vlavcheski F, Den Hartogh DJ, Giacca A, Tsiani E. Amelioration of High-Insulin-Induced Skeletal Muscle Cell Insulin Resistance by Resveratrol Is Linked to Activation of AMPK and Restoration of GLUT4 Translocation. Nutrients. 2020 Mar 27;12(4):914. doi: 10.3390/nu12040914. PMID: 32230718; PMCID: PMC7230755.

28 Zhang S, Zhou Y, Zhao L, Tian X, Jia M, Gu X, Feng N, An R, Yang L, Zheng G, Li J, Guo H, Fan R, Pei J. K-opioid receptor activation protects against myocardial ischemia-reperfusion injury via AMPK/Akt/eNOS signaling activation. Eur J Pharmacol. 2018 Aug 15;833:100-108. doi: 10.1016/j.ejphar.2018.05.043. Epub 2018 May 30. PMID: 29856969.

29 Mangano DT. Effects of acadesine on myocardial infarction, stroke, and death following surgery. A meta-analysis of the 5 international randomized trials. The Multicenter Study of Perioperative Ischemia (McSPI) Research Group. JAMA. 1997;277(4):325-332. doi:10.1001/jama.277.4.325

30 Beker MC, Caglayan B, Caglayan AB, Kelestemur T, Yalcin E, Caglayan A, Kilic U, Baykal AT, Reiter RJ, Kilic E. Interaction of melatonin and Bmal1 in the regulation of PI3K/AKT pathway components and cellular survival. Sci Rep. 2019 Dec 13;9(1):19082. doi: 10.1038/s41598-019-55663-0. PMID: 31836786; PMCID: PMC6910929.

31 Russell RR 3rd, Bergeron R, Shulman GI, Young LH. Translocation of myocardial GLUT-4 and increased glucose uptake through activation of AMPK by AICAR. Am J Physiol. 1999 Aug;277(2):H643-9. doi: 10.1152/ajpheart.1999.277.2.H643. PMID: 10444490.

\section{Figures}



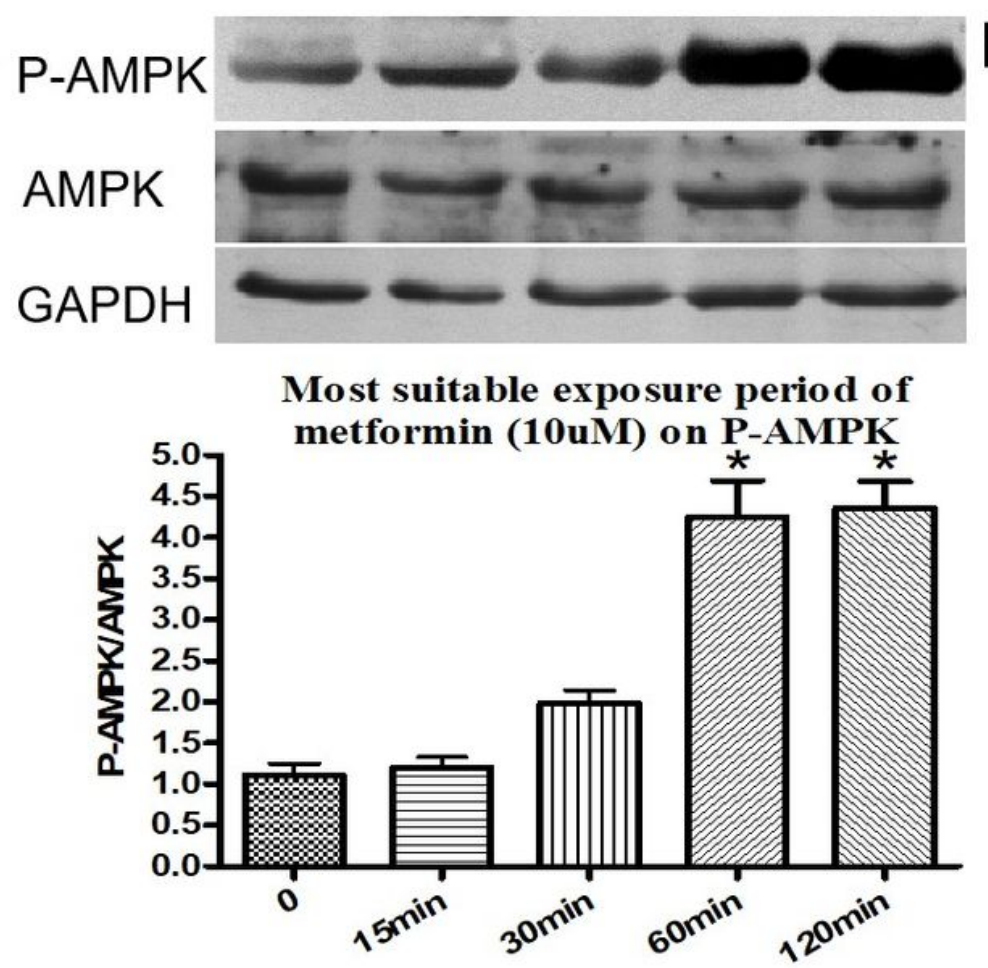

\section{$B_{\text {P-AMPK }}$}

AMPK

GAPDH

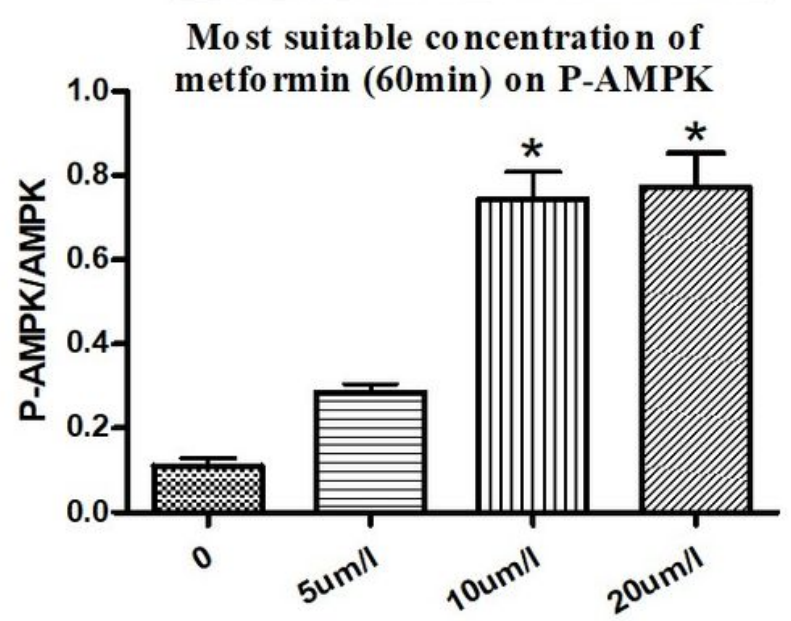

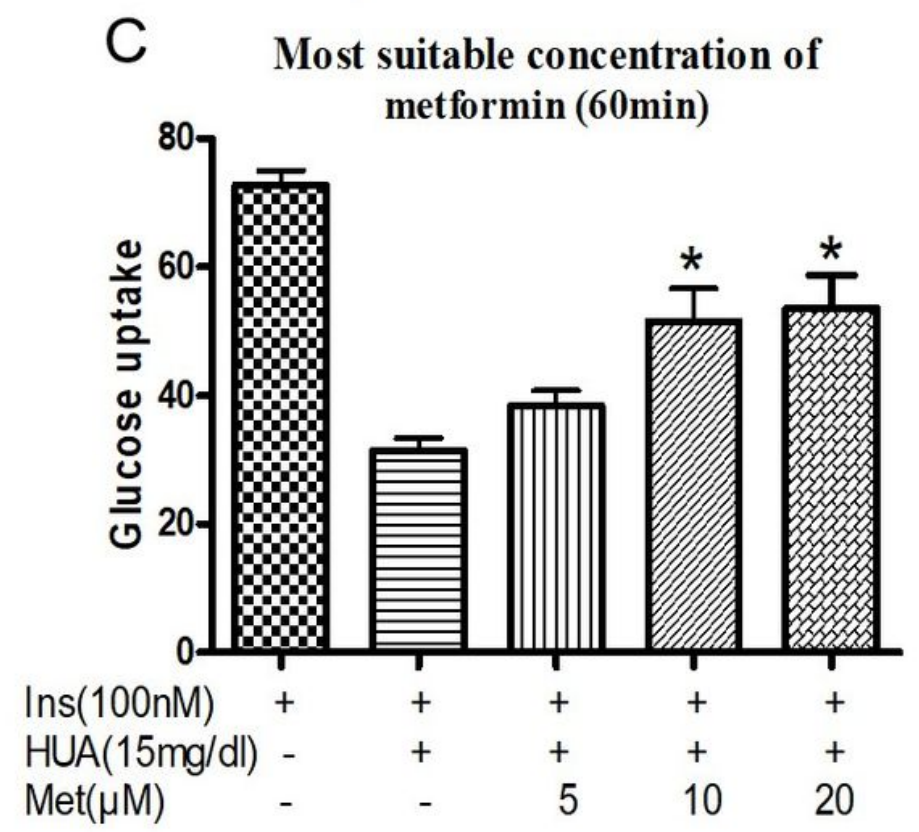

Figure 1

Effect of metformin on AMPK phosphorylation (A-B) and HUA-inhibited glucose uptake induced by insulin in primary cardiomyocytes (C). (A-B) Western blot analysis of phosphorylated and total AMPK levels. (C) Pretreated with metformin $(10 \mu \mathrm{mol} / \mathrm{L})$ for 60 minutes protected agains HUA (15mg/dl for 24 hours) inhibited 2-NBDG uptake induced by insulin in primary cardiomyocytes, as shown by flow cytometry analysis. $A,{ }^{*} P<0.05$ vs. 0,15 and 30 min. $B,{ }^{*} P<0.05$ vs. 0 and 5 uM. $C,{ }^{*} P<0.05$ vs. Ins+HUA. Data are mean $\pm S D$ from 4 separate experiments. Ins: insuln. HUA: high uric acid. Met: metformin. 2-NBDG: 2-[N-(7Nitrobenz-2-oxa-1,3-diazol-4-yl)amino]-2-deoxy-d-glucose. 


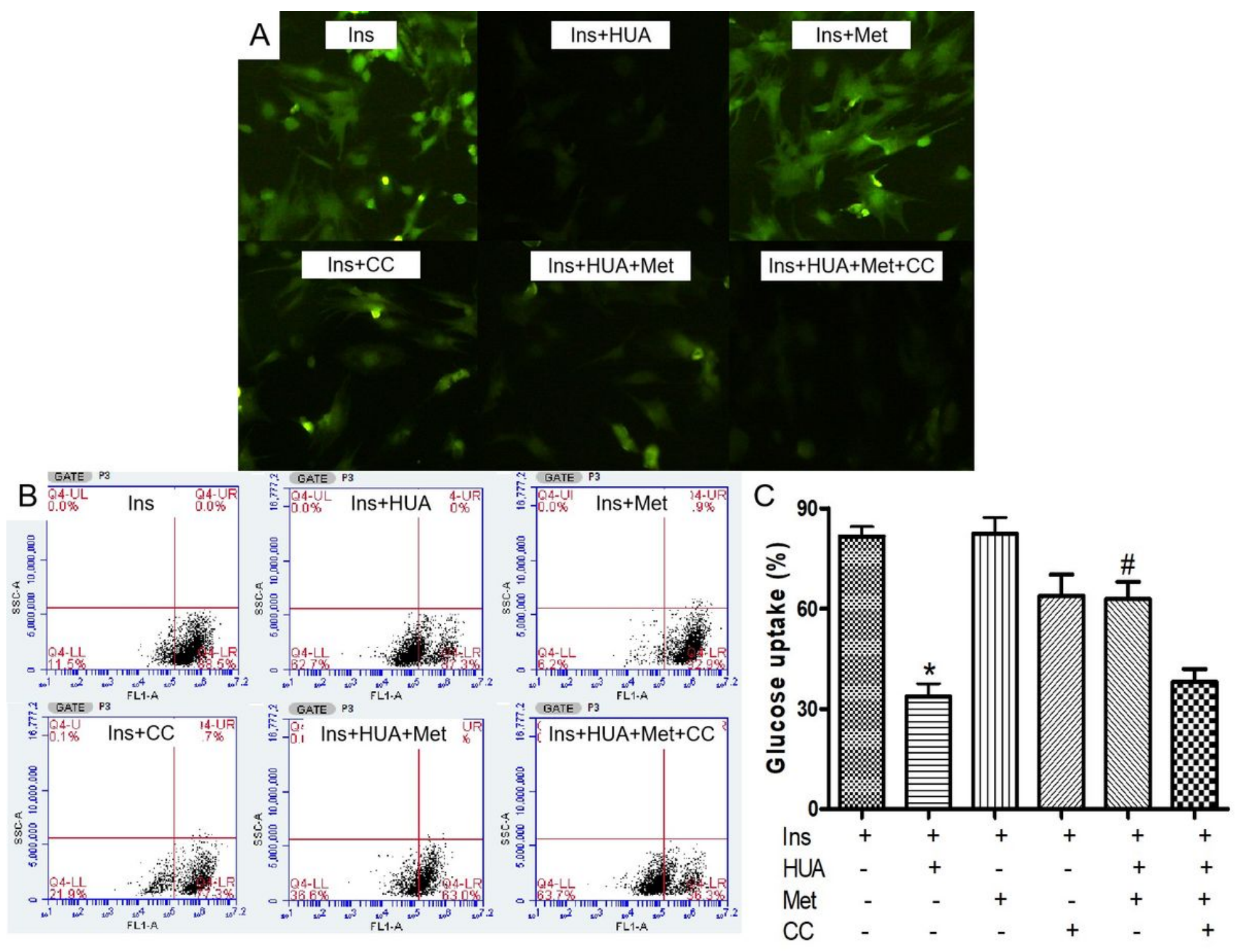

Figure 2

Metformin protected agains HUA inhibited 2-NBDG uptake induced by insulin via AMPK signal pathways in primary Cardiomyocytes. (A) 2-NBDG uptake assay detected by fluorescence microscopy. (B-C) 2-NBDG uptake assay detected by flow cytometry. ${ }^{*} \mathrm{P}<0.05 \mathrm{vs}$. Ins and Ins+HUA+Met, \#P<0.01 vs.

Ins+HUA+Met+CC. Data are mean \pm SD from 4 separate experiments. CC: compound C, an AMPK inhibitor. 


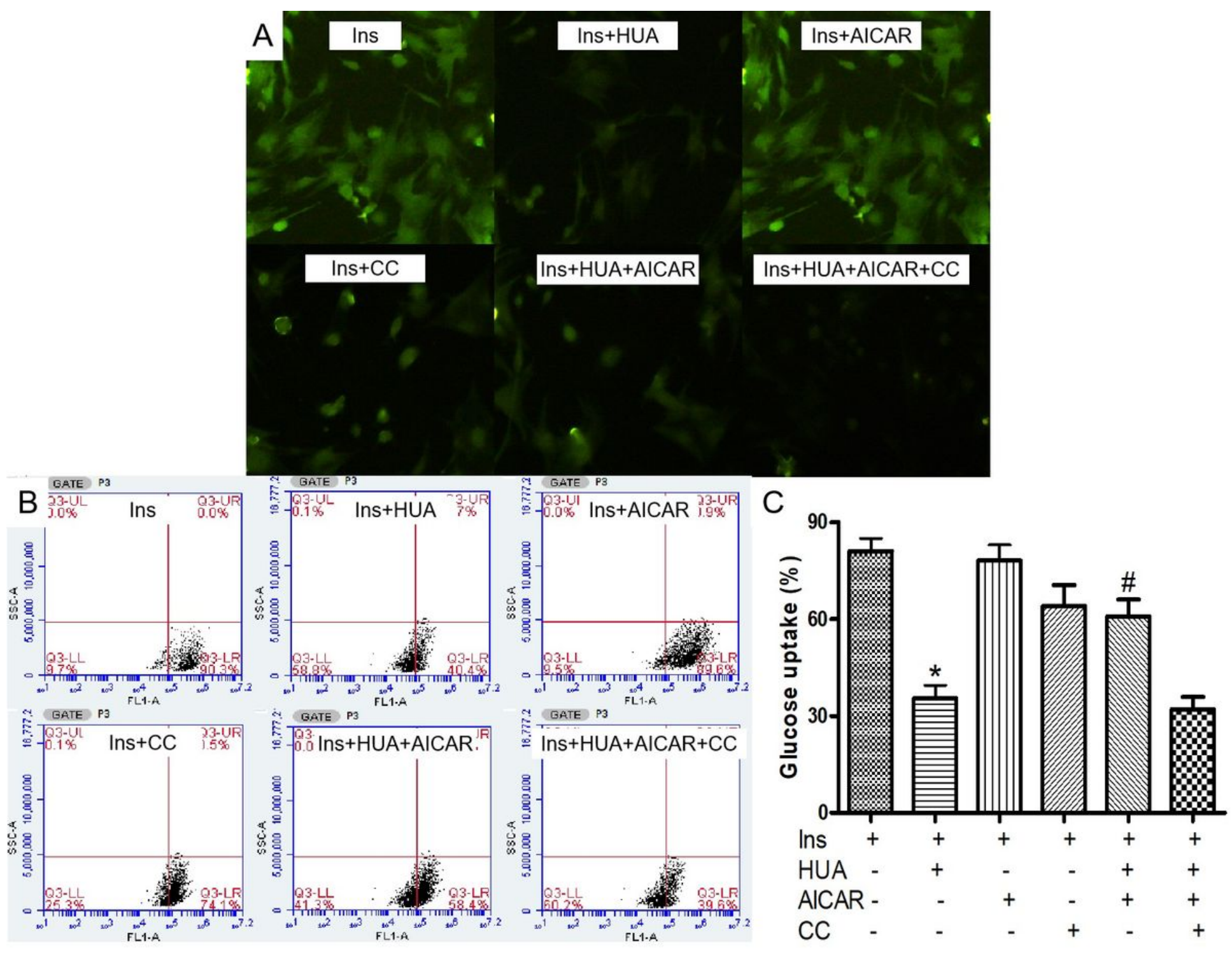

\section{Figure 3}

5-amino-4-imidazole-1- $\beta$-D-carboxamide ribofuranoside (AICAR, another AMPK activator) protected agains HUA inhibited 2-NBDG uptake induced by insulin via AMPK signal pathways in primary Cardiomyocytes. (A) 2-NBDG uptake assay detected by fluorescence microscopy. (B-C) 2-NBDG uptake assay detected by flow cytometry. ${ }^{*} \mathrm{P}<0.05$ vs. Ins and Ins+HUA+AICAR, \#P<0.01 vs. Ins+HUA+AICAR+CC. Data are mean $\pm S D$ from 4 separate experiments. 


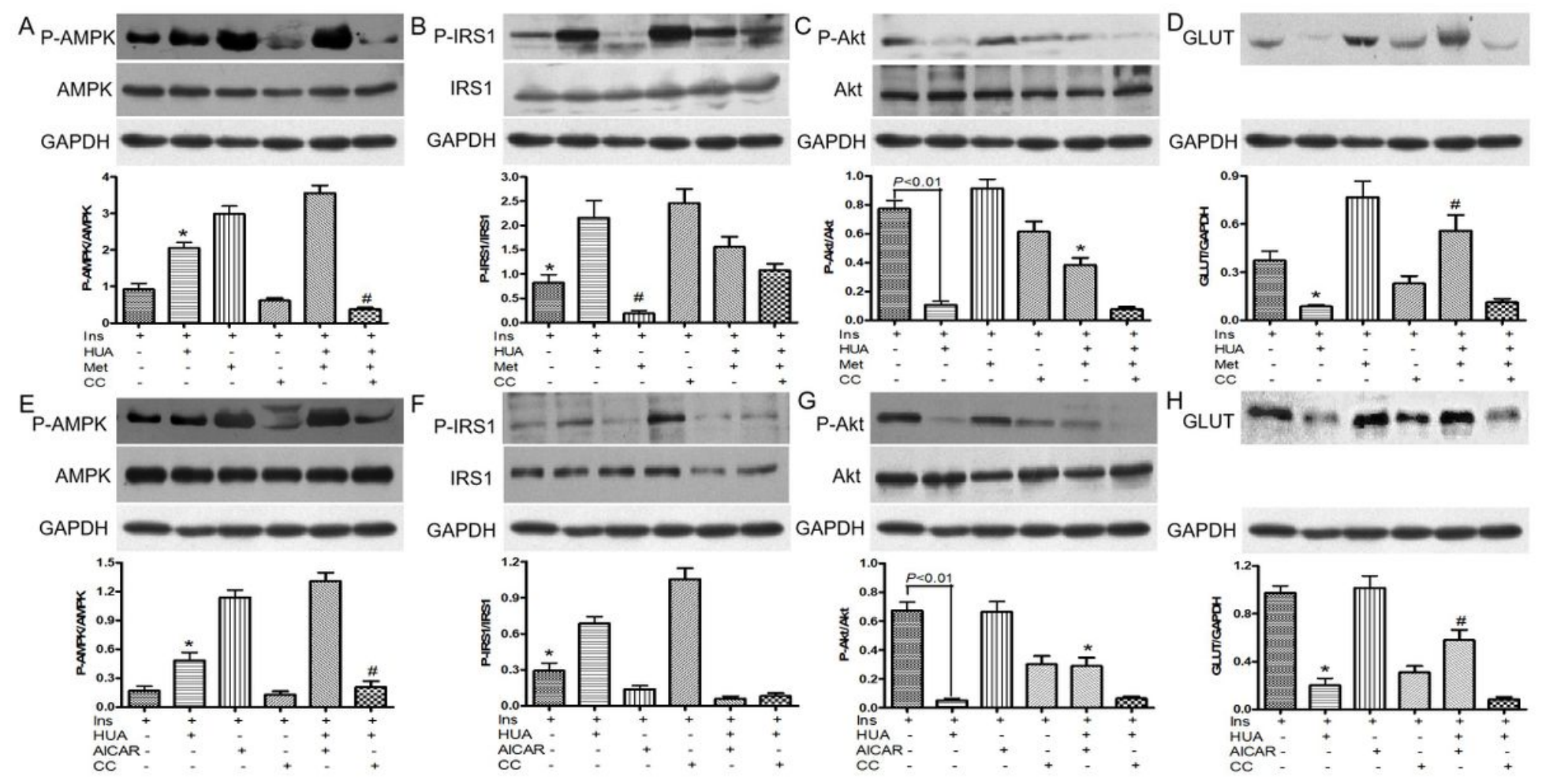

Figure 4

(A-D) Effect of merformin on HUA-induced/inhibited of phospho-AMPK (A), phospho-IRS1 (B), phosphoAkt (C) and GLUT (D) in primary Cardiomyocytes. A, ${ }^{\star} P<0.05$ vs. Ins and Ins+HUA+Met; \#P<0.01 vs. Ins+HUA+Met. $B,{ }^{*}<0.05$ vs. Ins+HUA and Ins+CC; $\# P<0.01$ vs. Ins+HUA+Met. $C,{ }^{*} P<0.05$ vs. Ins $+H U A$, Ins+Met and Ins+HUA+Met+CC. D, ${ }^{*}<<0.05$ vs. Ins and Ins+HUA+Met; $\# P<0.01$ vs. Ins+HUA. (E-H) Effect of AICAR on HUA-induced/inhibited of phospho-AMPK (E), phospho-IRS1 (F), phospho-Akt (G) and GLUT $(H)$ in primary Cardiomyocytes. $E,{ }^{*}<<0.05$ vs. Ins and Ins+HUA+Met; $\# P<0.01$ vs. Ins+HUA+Met. $F$, ${ }^{*} P<0.05$ vs. Ins+HUA and Ins+CC. G, ${ }^{*} P<0.05$ vs. Ins+HUA, Ins+Met and Ins+HUA+Met+CC. $H,{ }^{*} P<0.05$ vs. Ins and Ins+HUA+Met; \#P<0.01 vs. Ins+HUA. 
A

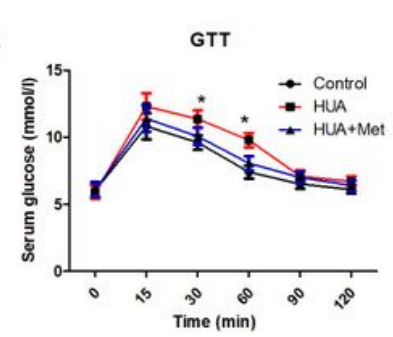

B

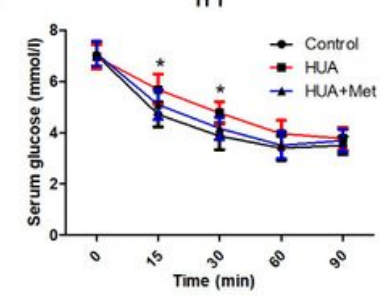

C

AMPK

GAPDH
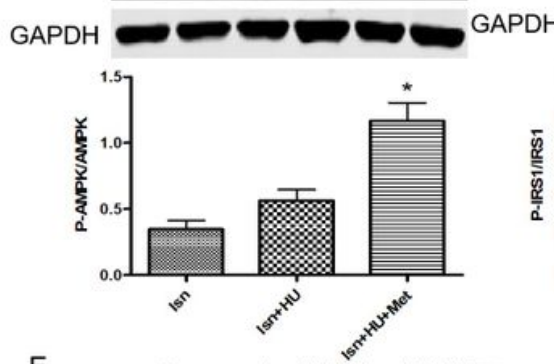

$E$
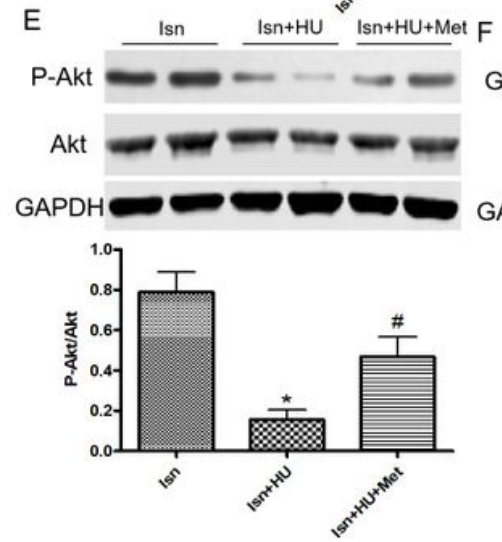
IRS1 $\frac{\text { Isn }}{\text { Isn+HU }} \stackrel{\text { Isn+HU+Met }}{-}$

Prist
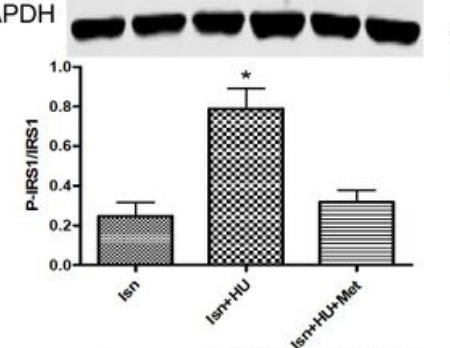
GLUT
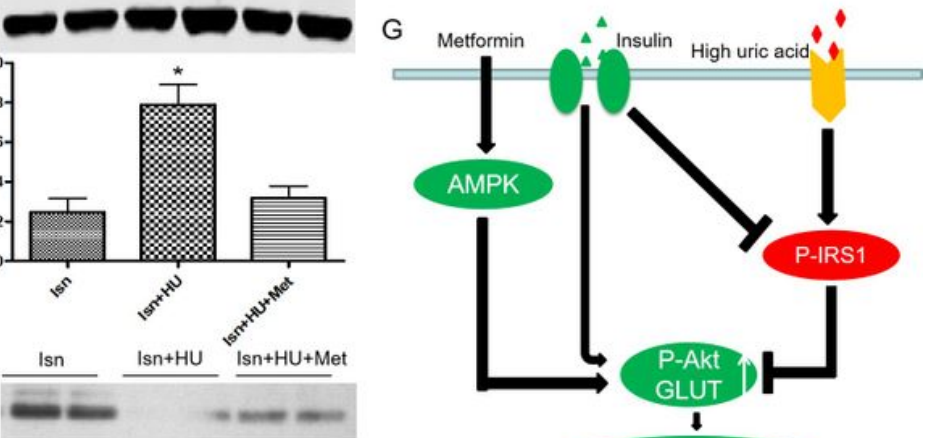

Glucose uptake
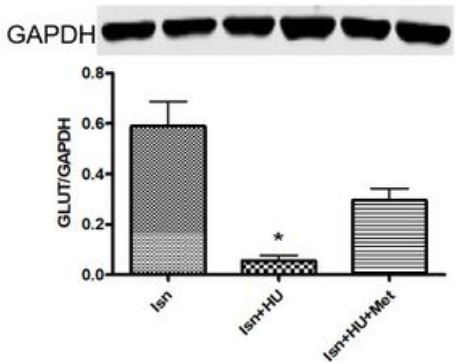

Figure 5

(A-B) Glucose tolerance test (GTT) (A) and insulin tolerance test (ITT) (B) in an acute hyperuricemic mice model. ${ }^{*} \mathrm{P}<0.05$ vs. Con and HU+Met. (C-F) Western blot analysis of phospho-AMPK (C), phospho-IRS1 (D), phospho-Akt (E) and GLUT (F) level in cardiac tissues. C, ${ }^{*} P<0.05$ vs. Ins+HU. D, ${ }^{*} P<0.05$ vs. Ins and Ins+HU+Met. $\mathrm{E}, * \mathrm{P}<0.05$ vs. Ins and Ins+HU+Met. $\mathrm{F},{ }^{*} \mathrm{P}<0.05$ vs. Ins and Ins+HU+Met. Data are mean $\pm S D$ from 4 separate experiments. (G) Schematic representation of how metformin ameliorates HUA-induced insulin resistance in cardiomyocytes. After treatment with HUA, which activates phosphorylation of IRS-1 (Ser307). This activity impairs AKT (Ser473) phosphorylation, for insulin resistance. After treatment with metformin, the phosphorylation of AMPK is increased. At the same time, this activity reverses the inhibition of HUA-induced AKT phosphorylation, which ameliorates HUA-induced insulin resistance. HU: hyperuricemia. Met: metformin. 